\title{
Estructura comunitaria de helmintos de perros vagabundos de San Juan de Lurigancho, Lima, Perú
}

\author{
José O. lannacone ${ }^{1}$ \\ Karina M. Cordova ${ }^{1}$ \\ Roberto V. Wong ${ }^{1}$
}

\begin{abstract}
Community structure of helminths in stray dogs from San Juan de Lurigancho, Lima, Peru. Thirty stray dogs Canis familiaris Linnaeus, 1758 were collected in San Juan de Lurigancho, Lima, Peru, between January and March 1997 and necropsied for helminths. Three species were collected: the cestodes Dipylidium caninum (Linnaeus, 1758) Railliet, 1892 and Taenia pisiformis (Bloch, 1780) and the nematode Toxocara canis (Werner, 1782) Stiles, 1905. Fifty per cent of the examined dogs showed one or two parasites. A total of $303(0-116)$ helminths were recovered and the intensity of infection was 30,3 . The Simpson index (C) for all parasite species was 0,414 , indicating a dominance by one species in the parasite community. The mean diversity in the infracommunities of $C$. familiaris was $\mathrm{H}^{\prime}=0,038$ and uniformity index of Pielou $\mathrm{J}=0,207$. Dipylidium caninum, $T$. pisiformis, and $T$. canis presented a prevalence of infection of $33,30 \%, 10 \%$ and $16,6 \%$ respectively. Dipylidium caninum presented the highest frequency and mean intensity of infection. Dipylidium caninum was the helminth with the highest dominance ( $94 \%$ ) whereas the lowest was due to $T$. pisiformis (4\%) and $T$. canis (2\%). Helminths were not related to the age of the hosts, prevalence and mean intensity of infection. Out of the 10 equally divided segments of the intestinal gut, D.caninum was found from the $6^{\text {th }}$ to the $9^{\text {th }}$ segment. $T$. canis preferred almost exclusively the $1^{\text {st }}$ segment while $T$. pisiformis was randomly distributed.

KEY WORDS. Canis familiaris, Dipylidium caninum, Toxocara canis, Taenia pisiformis, perros, Peru
\end{abstract}

A nivel mundial, el perro Canis familiaris Linnaeus, 1758 es el principal vertebrado empleado como mascota por el ser humano. Su helmintofauna parasitaria, es una de las mejores conocidas, incluso en un país neotropical como Brasil, se han registrado 28 especies diferentes de nemátodos (VICENTE et al. 1997).

HoIDA et al. (1998) indican el riesgo epidemiológico zoonótico para la salud humana de dos helmintos de importancia médica, Echinoccocus granulosus (Batsch, 1786) y Toxocara canis (Werner, 1782) Stiles, 1905 transmitidos por perros. El primero, es un céstodo que provoca hidatidosis y el segundo un nematodo, produce una enfermedad llamada "larva migrans visceral", ambos en el ser humano (WoOdRuFF 1975; OvergaAuW 1997, 1998). Otros parásitos de importancia veterinaria como Dipylidium caninum (Linnaeus, 1758) Railliet, 1892 y Taenia pisiformis (Bloch, 1780) pueden producir enfermedades diarreicas en $C$. familiaris

1) Laboratorio de Ecofisiologia, Area de Biodiversidad Animal, Universidad Nacional Federico Villarreal. Calle San Marcos 383 Pueblo Libre, Lima 21, Lima, Perú.

E-mail: joselorena@terra.com.pe 
(Turner \& Pegg 1977; Bugg et al. 1999; Wells \& HePper 1999). El adecuado conocimiento y manejo de las enfermedades parasitarias que afectan al perro y su impacto en la salud humana es un tópico altamente prioritario (STALLBAUMER 1987; CABRERA et al. 1996).

El análisis cuantitativo y la estructura biocenótica de los helmintos gastrointestinales en perros vagabundos y su relación con la edad y sexo del hospedero, han sido realizados en diferentes latitudes en todo el mundo (KAZACOS 1978; HASSAN 1982; Ajlouni et al. 1984; UgochuKwu \& EJimadu 1985b). En Perú, pocos trabajos sobre esta temática se han realizado (CABRERA \& ROJAS 1997; CABRERA et al. 1997; RojAS et al. 1997). Por lo que el objetivo de este trabajo es evaluar la estructura comunitaria de helmintos enteroparásitos a nivel del intestino delgado en perros vagabundos, en uno de los distritos más populosos de Lima, como San Juan de Lurigancho y su relación con algunos parámetros bioecológicos del hospederos, así como su preferencia a un sitio específico en el intestino delgado del mismo.

\section{MATERIAL Y MÉTODOS}

\section{Obtención del material biológico}

En la estación veraniega, desde el 15 de Enero al 30 de marzo de 1997, treinta individuos de Canis familiaris "perro" fueron examinados a nivel del intestino delgado para la busqueda de helmintos parásitos. Cada 25 días se capturaron 10 ejemplares de $C$. familiaris en el distrito de San Juan de Lurigancho, en coordinación con el centro de Salud de Lima Norte, Perú. Los perros vagabundos fueron llevados a la Unidad de Cirugía Experimental del Instituto de Salud del Niño (UCE-ISN) y se alojaron en sus respectivos caniles. Obteniéndose en total, 21 machos y 9 hembras. Los perros fueron codificados para facilitar el tratamiento de los datos. Los perros fueron alimentados diariamente con comida balanceada Ricocan ${ }^{\circledR}$, con $100 \mathrm{~g}$ por cada $8 \mathrm{k}$ de peso del perro. Los caniles fueron limpiados y desinfectados a diario con hipoclorito de sodio Clorox ${ }^{\circledR}$. Posteriormente a cada perro a ser operado se le anestesió, se le efectuó una laparotomía supraumbilical y luego se procedió a efectuar una enterectomía total, realizando hemostasia. Luego de retirar el intestino delgado, se procedió a cerrar por planos al perro para finalmente sacrificarlo sin dolor, según el código ético del Consejo de Organizaciones Internacionales de las Ciencias Médicas (COICM) sobre los experimentos con animales. Se determinó la edad de cada perro, mediante la observación de la dentadura, mediante las claves propuestas por CoGgins (1998). Se determinó el sexo, peso en $\mathrm{kg}$, talla en cm y longitud del intestino delgado en $\mathrm{cm}$ de cada perro.

\section{Análisis ecológico}

Las edades de los perros en meses se dividieron en cuatro grupos: 6,5 (3 a 12) meses, 24 (13-24) meses, 40,36 (25-48) meses y 70,5 (49-72) meses. Además, los perros machos presentaron una longitud entre $25-57 \mathrm{~cm}(43,95 \pm 9,73)$ y las hembras de $28-53(42,33 \pm 8,74)$. El intestino se dividió en 10 porciones iguales que se delimitaron con la ayuda de hilos anudados. Se denominó porción 1 a la más cercana al esfínter cardias y 10 a la más cercana al esfinter pilórico, siguiendo las 
recomendaciones de BusH \& HolmES (1986). Posteriormente se colocó el intestino en un frasco con formalina bufferada al $10 \%$ para su conservación y fijación de los helmintos adheridos a él. Se procedió a analizar individualmente cada una de las 10 partes con la ayuda del estereoscopio. Los céstodos se contabilizaron de acuerdo a los escolex encontrados en cada una de las 10 porciones examinadas. Todos los helmintos fueron procesados usando los procedimientos parasitológicos estandarizados para su identificación a nivel de especie. Los céstodos fueron fijados en AFA (Alcohol Formol Acido Acético), luego coloreados con carmín acético de Semichon y finalmente montados en bálsamo de Canadá. Los nematodos fueron fijados brevemente en ácido acético glacial, guardados en alcohol etílico al $70 \%$ con glicerina al $5 \%$ y finalmente clarificados y examinados en montajes húmedos en gelatina glicerinada. Los nemátodos por el número de individuos encontrados, se consideraron en conjunto los nemátodos machos y hembras. Material tipo de cada uno de los parásitos colectados fue depositado en la helmintoteca del Laboratorio de Ecofisiología de la Facultad de Ciencias Naturales y Matemáticas, Universidad Nacional Federico Villarreal.

La aproximación ecológica de las comunidades endoparasitarias metazoarias se realizaron a nivel de componentes e infracomunidades (EsCH et al. 1990). Con estos parámetros evaluados se procedió a calcular la prevalencia, intensidad y abundancia media de infección (BusH et al. 1997). La frecuencia de dominancia de los componentes de la fauna parasitaria se calculó de acuerdo a ZAR (1996).

Se emplearon algunos métodos estadísticos de asociación: el coeficiente de correlación de Spearman $\left(\mathrm{r}_{\mathrm{s}}\right)$ fue calculado para determinar una posible correlación entre la edad y la abundancia de los parásitos. El coeficiente de correlación de Pearson ( $\mathrm{r}$ ) se utilizó como una indicación de la relación de la edad y la prevalencia de parásitos, con previa transformación arcoseno de los datos de prevalencia (ZAR 1996). El efecto del sexo en la abundancia media se calculó usando la prueba de $t$ de student, previa evaluación de la homogeneidad de varianzas empleando la prueba de Levene y para el efecto en la prevalencia se usó el estadístico $X^{2}$ (FRANCO et al. 1995). Se utilizó nuevamente el coeficiente de correlación de Pearson (r) para relacionar la abundancia de las especies de helmintos presentes. El estadístico G se probó para determinar si existían diferencias significativas de la helmintofauna entre las diferentes porciones del intestino delgado. El nivel de significancia fue evaluado a $\alpha=0,05$ (DANIEL 1993). La terminología ecológica siguió los criterios de BUSH et al. (1997). El análisis ecológico incluyó solo parásitos con prevalencias mayores al 10\% (BusH et al. 1990). Especies comunes fueron definidas a aquellas especies con prevalencias mayores al $20 \%$. Se usó la terminología de especies centrales (presentes en más de $2 / 3$ de los hospederos), secundarias (presentes entre 1/3 a 2/3 de los hospederos) y satélites (presentes en menos de $1 / 3$ de los hospederos) propuesta por BUSH \& HoLMES (1986). Se empleo para el análisis comunitario de la diversidad de especies parásitas el índice de diversidad de Shannon Weaver $\left(\mathrm{H}^{\prime}\right)$ en base a la escala logarítmica en base 2. H' $=-\Sigma$ pilogpi, donde, $\log =$ logaritmo en base 2; $\mathrm{pi}=\mathrm{ni} / \mathrm{N}, \mathrm{ni}=$ número de individuos de cada especie y $\mathrm{N}=$ número total de individuos (ZAR 1996). Se calculó para cada infracomunidad parasitaria, el índice de uniformidad de Pielou (J) (Poulin 1995). El índice de Simpson (C) se usó para 
determinar la dominancia de la comunidad parasitaria, cuando $\mathrm{C} \geq 0,25$ (LUQUE \& Chavez 1999). Se empleo el paquete estadístico SPSS para window 95 para el cálculo de las pruebas estadísticas descriptivas e inferenciales.

\section{RESULTADOS}

Los ejemplares de $C$. familiaris examinados en este estudio consistieron en 21 machos (70\%) y 9 hembras (30\%). Por edades los perros se dividieron en cuatro categorías: ocho menores de 12 meses $(26,66 \%)$, tres entre 13 a 24 meses $(10 \%)$, 11 entre 25 a 48 meses $(36,66 \%)$ y ocho mayores de 49 meses $(26,66 \%)$.

De los 30 perros examinados, 15 no estuvieron infectados a nivel del intestino delgado (50\%), $12(40 \%)$ con una especie y $3(10 \%)$ con dos especies. El número modal por hospedero fue uno con un rango de uno a dos. Un total de 303 helmintos fueron recuperados $(0-116)\left(X^{2}=30,3\right)$. La tabla I muestra, los valores de prevalencia, frecuencia, intensidad y abundancia media de infección con sus rangos respectivos para las tres especies de helmintos a nivel del intestino delgado. Se observó con relación a la prevalencia de infección el siguiente orden decreciente: Dipylidium caninum $>$ Toxocara canis $>$ Taenia pisiformis. Para la intensidad y abundancia media de infección el orden decreciente fue: $D$. caninum $>T$. pisiformis $>T$. canis . La frecuencia de dominancia relativa fue para D. caninum, T. canis, y T. pisiformis de diez, tres y dos respectivamente. No se vio ninguna frecuencia de dominancia compartida. La figura 1 indica que $D$. caninum fue el helminto con mayor porcentaje de abundancia (94\%) y en menor cantidad $T$. pisiformis $(4 \%)$ y $T$. canis $(2 \%)$. El indice de Simpson, presentó una ligera dominancia, indicando que prevalece una especie en la fauna de helmintos en perros de San Juan de Lurigancho, Lima (Tab. II). La diversidad media de las infracomunidades y el índice de uniformidad o diversidad relativa media de Pielou fue relativamente bajo (Tab. II). De los tres helmintos analizados, una especie fue considerada secundaria: D. caninum, en cambio $T$. canis y $T$. pisiformis fueron especies satélites.

Tabla I. Helmintos colectados del intestino delgado de 30 ejemplares de Canis familiaris de Lima, Perú.

\begin{tabular}{|c|c|c|c|c|c|}
\hline \multirow{2}{*}{ Helmintos } & \multirow{2}{*}{ Prevalencia } & \multirow{2}{*}{ Frecuencia/Total } & \multicolumn{2}{|c|}{ Intensidad } & \multirow{2}{*}{$\begin{array}{c}\text { Abundancia } \\
\text { Media }\end{array}$} \\
\hline & & & Rango & Media & \\
\hline \multicolumn{6}{|l|}{ Cestoda } \\
\hline Dipylidium caninum * & 33,30 & 10 de 30 & 3 a 116 & 28,40 & 9,46 \\
\hline Taenia pisiformis * & 10,00 & 3 de 30 & $2 \mathrm{a} 7$ & 4,33 & 0,43 \\
\hline \multicolumn{6}{|l|}{ Nematoda } \\
\hline Toxocara canis ** & 16,60 & 5 de 30 & 1 a 2 & 1,20 & 0,20 \\
\hline
\end{tabular}

(*) Basado solamente en escólices presentes; $\left({ }^{\star *}\right)$ incluye en conjunto nemátodos hembras y machos.

No se encontraron diferencias estadísticamente significativas entre el sexo de $C$. familiaris y la abundancia media de $D$. caninum $(\mathrm{t}=0,72, \mathrm{P}=0,47, \mathrm{n}=30$ ), de $T$. pisiformis $(\mathrm{t}=0,83, \mathrm{P}=0,14, \mathrm{n}=30)$ y de $T$. canis $(\mathrm{t}=0,98, \mathrm{P}=0,33, \mathrm{n}=30)$. Tampoco, entre el sexo y la prevalencia de infección de $D$. caninum $\left(X^{2}=0,53, \mathrm{P}\right.$ 


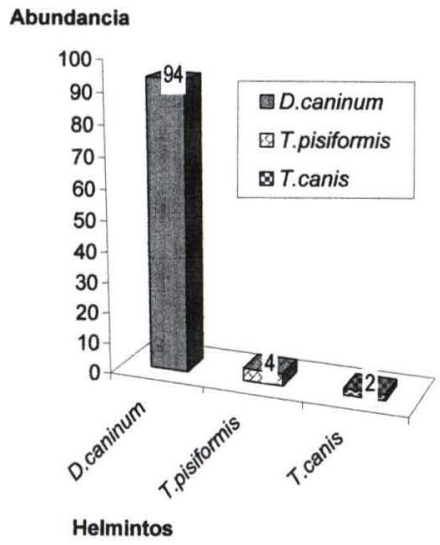

Fig. 1. Abundancia porcentual de tres helmintos en el intestino delgado de 30 ejemplares de C. familiaris de Lima, Perú.

Tabla II. Parámetros de las metacomunidades del intestino delgado de 30 ejemplares de Canis familiaris de Lima, Perú. (C) Indice de Simpson, ( $\left.\mathrm{H}^{\prime}\right)$ indice de diversidad, ( $\left.\mathrm{J}^{\prime}\right)$ indice de uniformidad de Pielou).

\begin{tabular}{lc}
\hline \multicolumn{1}{c}{ Indice } & Valor calculado \\
\hline C & 0,411 \\
H' & 0,038 \\
J' & 0,207 \\
H' para machos & 0,081 \\
J' para machos & 0,168 \\
H' para hembras & 0,166 \\
J' para machos & 0,348 \\
\hline
\end{tabular}

$=0,46, \mathrm{n}=30), T$. pisiformis $\left(X^{2}=0,69, \mathrm{P}=0,40, \mathrm{n}=30\right)$ y $T$. canis $\left(X^{2}=0,97, \mathrm{P}\right.$ $=0,32, \mathrm{n}=30$ ). No se encontró correlación entre la edad de $C$. familiaris y la abundancia media de D.caninum $\left(\mathrm{r}_{\mathrm{s}}=0,17, \mathrm{P}=0,34, \mathrm{n}=30\right.$ ), de $T$. pisiformis $\left(\mathrm{r}_{\mathrm{S}}=\right.$ $-0,04, \mathrm{P}=0,81, \mathrm{n}=30)$ y de $T$. canis $\left(\mathrm{r}_{\mathrm{s}}=-0,14, \mathrm{P}=0,45, \mathrm{n}=30\right)$. Tampoco se encontró correlación entre la edad y la prevalencia de infección de $D$. caninum $(\mathrm{r}=$ $0,86, \mathrm{P}=0,13, \mathrm{n}=4)$, de $T$. canis $(\mathrm{r}=-0,28, \mathrm{P}=0,71, \mathrm{n}=4)$ y de $T$. pisiformis $(\mathrm{r}=$ $0,22, \mathrm{P}=0,77, \mathrm{n}=4$ ) (Fig. 2).

La edad y el número de especies no se encontraron correlacionadas $(\mathrm{r}=0,06$, $\mathrm{P}=0,73, \mathrm{n}=30)$, lo mismo para la edad y el número de especímenes $(\mathrm{r}=0,04, \mathrm{P}=$ $0,87, \mathrm{n}=30$ ). Sin embargo, el número de especies y de especímenes se encontraron altamente correlacionados $(\mathrm{r}=0,93, \mathrm{P}=0,001, \mathrm{n}=30)$. El análisis de correlación de Pearson ( $r$ ) entre las intensidades de los tres helmintos mostró que no existe relación o asociación estadísticamente significativa entre sí (Tab. III). La tabla IV, indica una correlación de Pearson entre el peso versus talla, peso versus longitud del intestino, peso versus edad en meses y talla versus longitud del intestino, edad versus longitud del intestino. 


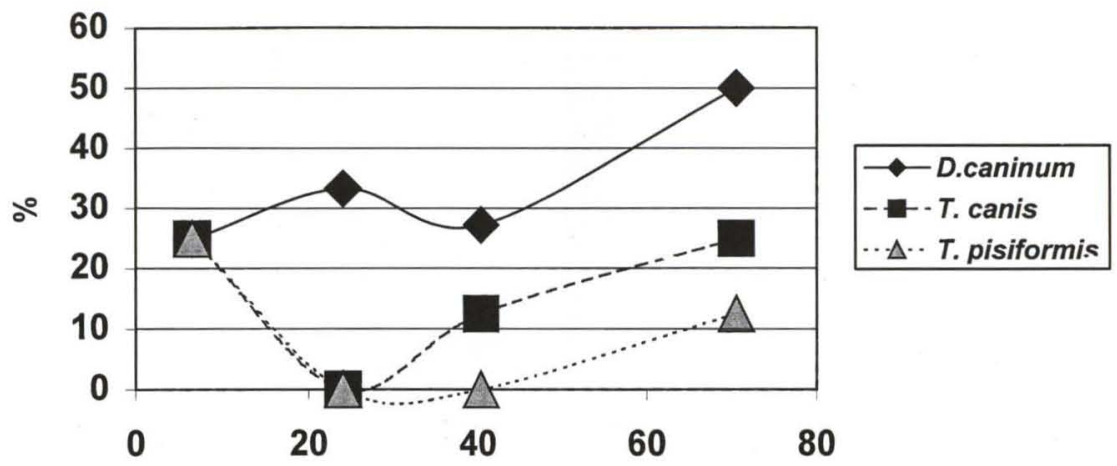

Edad en meses

Fig. 2. Prevalencia de infección (\%) de tres helmintos en perros vagabundos de Lima, Perú deacuerdo con las edades de los perros.

Tabla III. Matriz de correlación de Pearson entre los tres helmintos presentes en el intestino delgado en 30 ejemplares de C. familiaris de Lima, Perú. Valores superiores $=$ probabilidad $(P)$; valores inferiores $=$ coeficiente de correlación ( $r$ ).

\begin{tabular}{lcccc}
\hline \multicolumn{1}{c}{ Helminto } & & 1 & 2 & 3 \\
\hline Dipylidium caninum & 1 & - & 0,52 & 0,09 \\
Taenia pisiformis & 2 & $-0,12$ & - & 0,51 \\
Toxocara canis & 3 & $-0,35$ & $-0,12$ & - \\
\hline
\end{tabular}

Tabla IV. Matriz de correlación de Pearson entre el sexo, peso, talla, longitud del intestino delgado y edad en meses de Canis familiaris en Lima, Perú. Valores superiores $=$ probabilidad $(P)$; valores inferiores $=$ coeficiente de correlación (r).

\begin{tabular}{lcccccc}
\hline \multicolumn{1}{c}{ Helminto } & & 1 & 2 & 3 & 4 & 5 \\
\hline Sexo & 1 & - & 0,48 & 0,240 & 0,480 & 0,59 \\
Peso (g) & 2 & $-0,13$ & - & 0,001 & 0,006 & 0,02 \\
Talla (cm) & 3 & $-0,22$ & 0,70 & - & 0,010 & 0,12 \\
Longitud del intestino & 4 & $-0,13$ & 0,48 & 0,450 & - & 0,05 \\
Edad en meses & 5 & $-0,10$ & 0,40 & 0,290 & 0,350 & - \\
\hline
\end{tabular}

La tabla $\mathrm{V}$ señalan los valores comparativos de prevalencias de infección de $T$. canis en perros de diversas localidades geográficas del mundo, ubicándose en el 12 lugar de 19 valores de mayor a menor prevalencia. La tabla VI nos muestra valores comparativos de prevalencia de infección de $D$. caninum en 16 localidades geográficas del mundo, ubicándose el presente trabajo en el quinto lugar. La tabla VII nos indica valores comparativos de prevalencia de infección de T. pisiformis en siete localidades del mundo, ubicándose en el quinto lugar en el presente estudio. 
Tabla V. Valores comparativos de prevalencia de infección de Toxocara canis en perros.

\begin{tabular}{cll}
\hline Prevalencia (\%) & \multicolumn{1}{c}{ Localidad, Pais } & \multicolumn{1}{c}{ Referencia } \\
\hline 0,7 & Norte de Israel, Israel & HOIDA et al. (1998) \\
1,2 & Jordán & EL-SHEHABI et al. (1999) \\
3,0 & Nairobi, Kenia & WACHIRA et al. (1993) \\
7,0 & Rabat, Morocco & PANDEY et al. (1987) \\
11,8 & Poznan, Polonia & MIZGAJSKA \& LUTY (1998) \\
13,5 & Sierra Leona & HASSAN (1982) \\
14,9 & Calabar, Nigeria & UGOCHUKWU \& EJIMADU (1985a) \\
16,6 & Lima, Perú & ORIGINAL \\
17,9 & La Habana, Cuba & DUMENIGO et al. (1994) \\
18,3 & Indiana, EE.UU. & KAZACOS (1978) \\
19,0 & Irbid, Jordán & ABO-SHEHADA \& ZIYADEH (1991) \\
25,0 & Calabar, Nigeria & UGOCHUKWU \& EJIMADU (1985b) \\
26,6 & Halifax, Nueva Escocia & MALLOY \& EMBIL (1978) \\
30,0 & Sabah, Malaysia & MACADAMS et al. (1984) \\
32,0 & Pretoria, Sudáfrica & VERSTER (1979) \\
40,0 & Baghdad, Iraq & TARISH et al. (1986) \\
41,0 & Holanda & OVERGAAUW (1998) \\
41,1 & Zaria, Nigeria & DADA et al. (1979) \\
82,6 & Dublin, Irlanda & O'LORCAIN (1994) \\
\hline
\end{tabular}

Tabla VI. Valores comparativos de prevalencia de infección de Dipylidium caninum en perros.

\begin{tabular}{cll}
\hline Prevalencia (\%) & \multicolumn{1}{c}{ Localidad, País } & \multicolumn{1}{c}{ Referencia } \\
\hline 1,6 & Calabar, Nigeria & UGOCHUKWU \& EJIMADU (1985a) \\
1,9 & Calabar, Nigeria & UGOCHUKWU \& EJIMADU (1985b) \\
3,8 & Sierra Leona & HASSAN (1982) \\
13,2 & Florida, Uruguay & CABRERA et al. (1996) \\
16,3 & Indiana, EE.UU. & KAZACOS (1978) \\
17,3 & Norte de Israel, Israel & HOIDA et al. (1998) \\
19,0 & Jordán & AJLOUNI et al. (1984) \\
19,4 & Jordán & EL-SHEHABI et al. (1999) \\
19,7 & Pretoria, Sudáfrica & VERSTER (1979) \\
19,8 & Irbid, Jordania & ABO-SHEHADA \& ZIYADEH (1991) \\
20,0 & Sabah, Malasia & MACADAMS et al. (1987) \\
33,3 & Lima, Perú & ORIGINAL \\
40,4 & Rabat, Morocco & PANDEY et al. (1984) \\
45,0 & Nairobi, Kenia & WACHIRA et al. (1993) \\
50,0 & Baghdad, Iraq & TARISH et al. (1986) \\
97,8 & Zaria, Nigeria & DADA et al. (1979) \\
\hline
\end{tabular}

Tabla VII. Valores comparativos de prevalencia de infección de Taenia pisiformis en perros.

\begin{tabular}{cll}
\hline Prevalencia (\%) & \multicolumn{1}{c}{ Localidad, Pais } & \multicolumn{1}{c}{ Referencia } \\
\hline 2,0 & Norte de Israel, Israel & HOIDA et al. (1998) \\
8,0 & Jordania & AJLOUNI et al. (1984) \\
10,0 & Lima, Perú & ORIGINAL \\
10,8 & Jordania & EL-SHEHABI et al. (1994) \\
12,3 & Rabat, Morocco & PANDEY et al. (1987) \\
44,1 & Irbid, Jordania & ABO-SHEHADA \& ZIYADEH (1991) \\
60,0 & Baghdad, Iraq & TARISH et al. (1986) \\
\hline
\end{tabular}


La figura 3 nos muestra que existen preferencias de las comunidades helmínticas en perros vagabundos del distrito de San Juan de Lurigancho, Lima-Perú a las porciones VI al IX del intestino delgado $(\mathrm{G}=49,77 ; \mathrm{P}<0,05$, g.l. $=9)$. D.caninum mostró preferencias a localizarse entre la VI a IX porción del intestino delgado $(\mathrm{G}=$ 40,11; P < 0,05; g.I. = 9). T. canis se localizó preferentemente en la I porción $(\mathrm{G}=$ 17,80, P<0,05, g.l. =9) y $T$. pisiformis no se localizó preferentemente en ninguna de las diez porciones intestinales $(\mathrm{G}=6,53, \mathrm{P}>0,05, \mathrm{~g} . \mathrm{l} .=9)(\mathrm{Tab} . \mathrm{VIII})$.

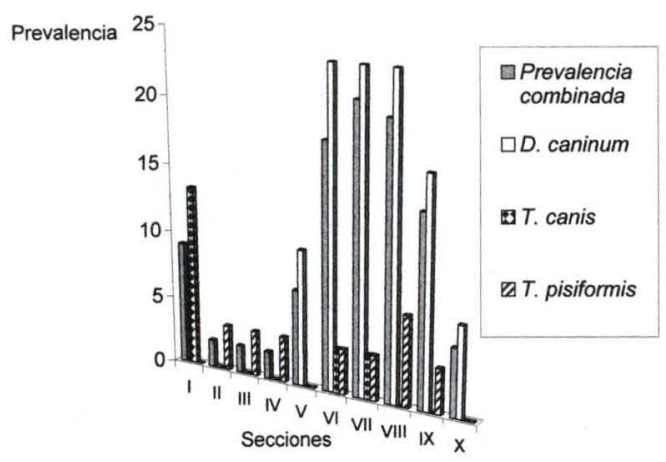

Fig. 3. Prevalencia de tres helmintos parásitos según cada una de las secciones del intestino delgado de $C$. familiaris de Lima, Perú.

Tabla VIII. Frecuencia de helmintos en cada una de las 10 porciones del intestino delgado en 30 ejemplares de Canis familiaris de Lima, Perú.

\begin{tabular}{lllllllllll}
\hline \multicolumn{1}{c}{ Helminto } & I & II & III & IV & V & VI & VI & VII & IX & X \\
\hline Dipylidium caninum & 0 & 0 & 0 & 0 & 3 & 7 & 7 & 7 & 5 & 2 \\
Taenia pisiformis & 0 & 1 & 1 & 1 & 0 & 1 & 1 & 2 & 1 & 0 \\
Toxocara canis & 4 & 0 & 0 & 0 & 0 & 1 & 1 & 0 & 0 & 0 \\
\hline
\end{tabular}

\section{DISCUSIÓN}

STOCK \& HolmES (1988), consideran dos tipos de comunidades parasitarias: interactivas (con especies centrales y evidencias de relaciones interespecíficas) y aisladas (con ausencia de especies centrales y de relaciones interespecíficas), pero no en forma dicotómica, en el cual serían clasificados todos los tipos de comunidades parasitarias, sino en una forma continua, formada por un conjunto de comunidades con características intermedias entre el tipo interactivo y aislada. En el presente caso, las comunidades enteroparasitarias de los perros vagabundos de Lima, Perú, carecen de especies centrales y ninguna de las tres especies se encontraron asociadas, mostrando una proximidad a las comunidades aisladas. Sin embargo, los resultados del análisis de las comunidades de helmintos intestinales para perros de otras latitudes, muestran en general, características de comunidades intermedias entre interactivas y aisladas, debido al alto número de especies secundarias (STALLBAUMER 1987; ABO-SHEHADA \& ZIYADEH 1991; BUGG et al. 1999). 
En el presente estudio, se encontró solo el $50 \%$ de los hospederos parasitados, encontrándose diferencias con valores más altos con lo reportado por EL-SHEHABI et al. (1999) que mostró una prevalencia combinada de 70,3\%, UGOSHUKWU \& EJMADU (1985a) de $86,9 \%$ y KAZACOS (1978) de 86,5\%. Esto se explicaría, debido a que las comunidades parasitarias de los hospederos sujetos a la influencia de frecuentes alteraciones ambientales, pueden sufrir variaciones en su riqueza y estructura comunitaria (Cislo \& CAIRA 1993; BRAsil-SATo \& PAVANELli 1999). El céstodo $E$. granulosus, no se detectó en el presente estudio, esto armoniza con lo encontrado por PANDEY et al. (1987) que indica que esta especie de céstodo no fue detectada en perros urbanos en Morocco (Africa). Por otro lado, Ancylostoma caninum (Ercolani, 1859), tampoco se encontró en el presente estudio, siendo una de las especies de nematodos más prevalentes (> 70\%) en numerosos estudios a nivel mundial (KAZACOS 1978; MACADAMS et al. 1984; UGOCHUKWU \& EIMADU 1985a,b).

La riqueza parasitaria en el presente estudio fue de 3 , sin embargo a nivel mundial se han encontrado de 4 a 12 diferentes especies de helmintos enteroparásitos. Numéricamente, los céstodos (2 especies) estuvieron mejor representados que los nematodos (1 especie); sin embargo, otros reportes a nivel mundial, indican que los nematodos fueron el grupo de mayor riqueza parasitaria (DADA et al. 1979; VERSTER 1979; HASSAN 1982; KAZACOS 1978; AJlOUNI et al. 1984; MACADAMS et al. 1984; TARISH et al. 1986; PANDEY et al. 1987; WACHIRA et al. 1993). La infección monoespecífica fue lo más común; sin embargo otros autores señalan la predominancia de una infección cocurrente con 2 o 3 especies (MACADAMS et al. 1984).

No existió influencia de la edad de los animales en la abundancia media y en la prevalencia de infección en ninguna de las tres especies de helmintos parásitos (Fig. 2). DuMENigo et al. (1994) mostró que la edad de los perros influyó en la prevalencia de infección de T. canis. MALLOY \& EMBIL (1978) mostraron que la prevalencia de $T$. canis fue mayor en perros de menor edad $(56,1 \%)$ que en animales de mayor edad (11,9\%) (MACADAMS et al. 1984; PANDEY et al. 1987; RICHARDS et al. 1993). OVERGAAUw (1997) muestra que el peculiar ciclo biológico de T. canis por su ruta de transmisión transplacentaria y transmamarial, favorecería el incremento del parasitismo en perros de menor edad (UGOCHUKWU \& EJIMADU 1985b). MACADAMS et al. (1984) indicó que la prevalencia de $D$. caninum fue independiente de la edad del perro. Este patrón fue acá observado.

La influencia del sexo de los hospederos, es un aspecto que viene siendo muy citado en la literatura y minimizado en los trabajos sobre análisis comunitario de endoparásitos en vertebrados (JANOVY et al. 1992; Poulin 1996a b). El que no existan diferencias cuantitativas en relación al sexo del hospedero, es considerado un reflejo de ausencia de diferencias en la biología y la dinámica poblacional entre hospederos machos y hembras. El presente trabajo, armoniza con lo encontrado por UgOCHUKWU \& EJIMAdu (1985a), GUBERTI et al. (1993), DUMENINGO et al. (1994), O'LorCAIN (1994) y WeLlS y HePPER (1999). Sin embargo, algunos autores han registrado una mayor prevalencia total combinada (KAZACOS 1978), solo por nematodos (PANDEY et al. 1987) y solo por D. caninum (AJLOUNI et al. 1984) en perros machos que en hembras. Estos autores, explican que ligeras diferencias en el comportamiento de los perros hembras, en la dieta y principalmente 
en el grado de saneamiento del hospedero, pudieran influenciar diferencias en la estructura comunitaria, debido al incremento de Ctenocephalides canis (Curtis, 1826), hospedero intermediario de D. caninum (PUGH \& Moorhouse 1985).

Los cánidos, entre ellos $C$. familiaris, es considerado uno de los hospederos definitivos más importantes para T. pisiformis (TIEKOTTER 1985). Los lagomorfos, entre ellos, Oryctolagus cuniculi Linnaeus, 1758 son considerados los más importantes hospederos intermediarios para Taenia pisiformis. Se han reportado prevalencias del 95\% en coyotes de Kansas (Holmes \& PODESTA 1968; MACKIEWICZ 1988). La infección por T. canis, puede ser más alta en ambientes urbanos que rurales (MizGAJSKA \& LUTY 1998). Toxocara canis exhibe dos modos primarios de infección: transmisión directa y por vía de un hospedero intermediario, el cual es usualmente un roedor (RICHARDS et al. 1993).

\begin{abstract}
AGRADECIMIENTOS. A Eduardo Esqueche M., Jefe de la Unidad de Cirugía Experimental del Instituto de Salud del Niño por el apoyo y asesoría en dicha unidad. A Juan Monteverde M., Jefe del Centro de Salud del Rímac, a cargo del área de Salud ambiental del Cono Este, quien nos facilitó la adquisición y transporte de los perros. A Henry Hernández I., de la Unidad de Cirugía Experimental del Instituto de Salud del Niño, por su apoyo profesional. A los laboratorios de la Universidad Nacional Federico Villarreal por el apoyo en la presente investigación.
\end{abstract}

\title{
REFERENCIAS BIBLIOGRAFICAS
}

ABo-SHEhADA, M.N. \& Y. ZIYADEII. 1991. Prevalence of endoparasites in dogs faecal deposits in Jordan. Jour. Helminthol. 65: 313-314.

AJlounl, A.Q.; E.K. SAliBA \& A.M. Disı. 1984. Intestinal cestodes of stray dogs in Jordan. Ztschr. Parasitenkd. 70: 203-210.

Brasil-Sato, M.C. \& G.C. Pavanelli. 1999. Ecological and reproductive aspects of Neoechinorhynchus pimelodi Brasil-Sato \& Pavanelli (Eoacanthocephala, Neoechinorhynchidae) of Pimelodus maculatus Lacépede (Siluroidei, Pimelodidae) of Sáo Francisco River, Brazil. Revta bras. Zool. 16: 73-82.

BugG, R.J.; I.D. ROBERTSON; A.D. Elliot \& R.C. ThOMPSON. 1999. Gastrointestinal parasites of urban dogs in Perth, Western Australia. Vet. Jour. 157: 295-301.

BUSH, A.O. \& J.C. HoLmES. 1986. Intestinal helminths of lesser scaup ducks: an interactive community. Can. Jour. Zool. 64: 142-152.

BUSH, A.O.; J.M. AHO \& C.R. KENNEDY. 1990. Ecological versus phylogenetic determinants of helminth parasite community richness. Evol. Ecol. 4: 1-20.

Bush, A.O; K.D. LAFFERTY; J.M. LOTZ \& A.W. Shostak. 1997. Parasitology meets ecology on its own terms: Margolis et al. revisited. Jour. Parasitol. 83: 575-583.

Cabrera, P.A.; S. Parietti; G. Haran; U. Benavidez; S. Lloyd; G. Perera; S. Valledor; M.A. GEMmELl \& T. BotTo. 1996. Rates of reinfection with Echinococcus granulosus, Taenia hydatigena, Taenia ovis and other cestodes in a rural dog population in Uruguay. Int. Jour. Parasitol. 26: 79-83.

Cabrera, R. \& R. Rojas. 1997. Un caso de difilobotriasis en la provincia de Chincha, Ica. Bol. Per. Parasitol. 12: 81 .

Cabrera, R.; R. Rojas \& M. Dávalos. 1997. Corynosoma obtuscens Lincicorne, 1943 (Acanthocephala: Polymorphidae) en Canis familiaris de la Ciudad Chincha, Ica. Bol. Per. Parasitol. 12: 82. 
Cislo, P.R. \& J.N. Caira. 1993. The parasite assemblage in the spiral intestine of the shark Mustelus canis. Jour. Parasitol. 79: 886-899.

Coggins, J.R. 1998. Effect of Season, Sex, and Age on Prevalence of Parasitism in Dogs from Southeastern Wiscosin. Jour. Helminthol. Soc. Wash. 65: 219-224.

DADA, B.J.; D.S. ADEGBoye \& A.N. MOHAMmED. 1979. A survey of gastro intestinal helminth parasites of stray dogs in Zaria, Nigeria. Vet. Rec. 104: 145-146.

DANiel, W.W. 1993. Bioestadística: Base para el análisis de las ciencias de la salud. México, D.F., Limusa, $3^{\text {ra }}$ ed., $667 \mathrm{p}$.

Dumenigo, B.; N. Lau \& J.R. Bravo. 1994. Prevalence of Toxocara canis in dogs in the City of Habana. Rev. Cubana Med. Trop. 46: 99-102.

El-Shehabi, F.S., S.K. Abdel-Hafez \& S.A. Kamhaw. 1999. Prevalence of intestinal helminths of dogs and foxes from Jordan. Parasitol. Res. 85: 928-934.

Esch, G.W.; A.W. Shostak; D.J. Marcogliese \& T.M. Goater. 1990. Pattern and processes in helminth parasite communities, p. 1-19. In: G. Esch; A.O. BusH \& J. Aно (Eds). Parasite communities: patterns and processes. New York, Chapman \& Hall, 335p.

Franco, L.J.; G. de la Cruz; A. Rocha; N. Navarrete; G. Flores; E. Kato; S. Sánchez; L. Abárca \& C.M. BEDIA. 1995. Manual de ecología. México, D.F., Trillas, $2^{\text {da }}$ ed., 266p.

GUBERTI, V.; L. STANCAMPIANO \& F. FRANCISCI. 1993. Intestinal helminth parasite community in wolves (Canis lupus) in Italy. Parasitologia 35: 59-65.

HASSAN, I.C. 1982. Gastrointestinal helminth parasites of dogs in the Western Area-Freetown (Sierra Leone). Beitr. Trop. Landwirtsh Veterinarmed. 20: 401-407.

Holmes, P.R. \& R. PODESTA. 1968. The helminths of wolves and coyotes from the forested regions of Alberta. Can. Jour. Zool. 46: 1193-1204.

Hoida, G.; Z. Greenberg; M. Furth; Y. Malsha; P.S. Craig; P.M. Schantz; R. Sneir \& J. El-On. 1998. An epidemiological survey of Echinococcus granulosus and other helminths in animal population in northern Israel. Jour. Helminthol. 72: 127-131.

JanOVY, J.; R.E. Clopton \& T.J. PERCIVAL. 1992. The roles of ecological and evolutionary influences in providing structure to parasite species assemblages. Jour. Parasitol. 78: 630-640.

KAZACOS, K.R. 1978. Gastrointestinal helminths in dogs from a humane shelter in Indiana. Jour. Amer. Vet. Med. Assoc. 173: 995-997.

Luque, J.L. \& N.D. Chavez. 1999. Ecología da comunidade de metazoarios parasitos da anchova Pomatomus saltator (Linnaeus) (Osteichthyes, Pomatomidae) do litoral do estado do Rio de Janeiro, Brasil. Revta bras. Zool. 16: 711-723.

MacAdams, I.; D. Gudan; D.V. TimbS; H.R. UrquharT \& M.M. Sewell. 1984. Metazoan parasites of dogs in Sabah, Malaysia. Trop. Anim. Health Prod. 16: 34-.38.

MAcKıewicz, J.S. 1988. Cestode transmission patterns. Jour. Parasitol. 74: 60-71.

MALLOY, W.F. \& J.A. EMBIL. 1978. Prevalence of Toxocara spp. and other parasites in dogs and cats in Halifax, Nova Scotia. Can. Jour. Comp. Med. 42: 29-31.

Mizgajska, H. \& T. LuTY, 1998. [Toxocariasis in dogs and contamination of soil with Toxocara spp. egss in the Poznan region]. Przegl. Epidemiol. 52: 441-446.

O'LorCaIn, P. 1994. Epidemiology of Toxocara spp. in stray dogs and cats in Dublin, Ireland. Jour. Helminthol. 68: 331-336.

Overganuw, P.A. 1997. Aspects of Toxocara epidemiology: Toxocara in dogs and cats. Crit. Rev. Microbiol. 23: 233-251.

1998. Toxocara infections in dogs and cats and public health implications. Vet. Q. 1: S97-98.

Pandey, V.S.; A. DakKaK \& M. Elmamoune. 1987. Parasites of stray dogs in the Rabat region, Morocco. Amer. Trop. Med. Parasitol. 81: 53-55.

Poulin, R. 1995. Phylogeny, ecology, and richness of parasite communities in vertebrates. Ecol. Monogr. 65: 283-302.

1996a. The evolution of life hisotry strategies in Parasitic animals. Adv. Parasitol. 37: 107-134. 
1996b. Sexual inequalities in helminth infections: a cost of being a male. Amer. Nat. 147: 287-295.

Pugh, R.E. \& D.E. Moorhouse. 1985. Factors affecting the development of Dipylidium caninum in Ctenocephalides felis felis (Bouche, 1835). Ztschr. Parasitenkd 71: 765-775.

RichardS, D.T.; S. HARRIS \& J.W. LEWIS. 1993. Epidemiology of Toxocara canis in red foxes (Vulpes vulpes) from urban areas of Bristol. Parasitology 107: 167-175.

Rojas, R; M. DÁvalos \& R. CabreRa. 1997. Prevalencia de Corynosoma obtuscens (Acanthocephala) en Canis familiaris de las provincioas de Chincha y Pisco, 1996. Bol. Per. Parasitol. 12: 82.

Stallbaumer, M. 1987. The prevalence and epidemiology of cestodes in dogs in Clwyd, Wales, II. Hunting dogs. Ann. Trop. Med. Parasitol. 81: 42-47.

STOCK, T.M. \& J.C. Holmes. 1988. Functional relationships and microhabitat distributions of enteric helminths of grebes (Podicipedidae): the evidence for interactive communities. Jour. Parasitol. 74: 214-227.

TARISH, J.H.; I.M. AL SAQUR; AL-ABBASSY \& F.S. KADHIM. 1986. The prevalence of parasitic helminths in stray dogs in the Baghdad area, Iraq. Ann. Trop. Med. Parasitol. 80: 329-331.

Tiekotter, K.L. 1985. Helmith species diversity and biology in the bobcat, Lynx rufus (Schreber), from Nebraska. Jour. Parasitol. 71: 227-234.

TuRner, T. \& E. PegG. 1977. A survey of patent nematode infestations in dogs. Vet. Rec. 100: 284-285. Ugochukwu, E.I. \& K.N. EjImAdu. 1985a. Studies on the prevalence of gastro-intestinal helminths of dogs in Calabar, Nigeria. Int. Jour. Zoonoses. 12: 214-218.

. 1985b. Comparative studies on the infestation of three different breeds of dogs by gastro-intestinal helminths. Int. Jour. Zoonoses. 12: 318-322.

Verster, A. 1979. Gastrointestinal helminths of somestic dogs in the Republic of south Africa. Onderstepoort Jour. Vet. Res. 46: 79-82.

Vicente, J.J.; H.O. Rodrigues; D.C. Gomes \& R.M. Pinto. 1997. Nematóides do Brasil. Parte V: Nematóides de Mamíferos. Revta bras. Zool. 14: 1-452.

Wachira, T.M.; M. Sattran; E. Zeyle \& M.K. NJEnga. 1993. Intestinal helminths of public health importance in dogs in Nairobi. East Afr. Med. Jour. 70: 617-619.

Wells, D.L. \& P.G. HePper. 1999. Prevalence of diseases in dogs purchased from an animal rescue shelter. Vet. Rec. 144: 35-38.

Woodruff, A.W. 1975. Toxocara canis and other nematodes transmitted from dogs to man. Br. Vet. Jour. 131: 627-637.

ZAR, J.H. 1996. Biostatistical Análisis. New Jersey, Pratence-Hall, Inc., $3^{\text {rd }}$ ed., 662p.

Recebido em 18.V.2000; aceito em 29.VI.2001. 